\title{
Bicuspid Aortic Valve Regurgitation: Quantification of Anatomic Regurgitant Orifice Area by 3D Transesophageal Echocardiography Reconstruction
}

\author{
Alessandro Malagoli, M.D., Andrea Barbieri, M.D., and Maria Grazia Modena, M.D., F.E.S.C. \\ Department of Cardiology, Policlinico Hospital, Modena and Reggio Emilia University, \\ Modena, Italy
}

(ECHOCARDIOGRAPHY, Volume 25, August 2008)

A 53-year-old man presented for routine clinical assessment to evaluate a longstanding systemic hypertension. Clinical exam was

Address for correspondence and reprint requests: Alessandro Malagoli, M.D., Department of Cardiology, Policlinico Hospital, Modena and Reggio Emilia University. Via del Pozzo 71, 41100 Modena, Italy. Fax: +39-059-4224323; E-mail: ale.mala@tiscali.it unremarkable. Transthoracic echocardiography (TTE) showed moderate aortic root dilatation (at sinus of Valsalva: $44 \mathrm{~mm}$ ) compared to normal references for age and body surface area and an eccentric regurgitation of aortic valve. Two-dimensional (2D) transesophageal echocardiography (TEE) and threedimensional (3D) TEE acquisitions were obtained with the same ultrasound unit that
Figure 1. A. 2D TTE $40^{\circ}$ : Bicuspid aortic valve with right and left cusp fusion. B. $2 D$ TEE $99^{\circ}:$ Measures of aortic root. C. $3 D$ TEE reconstruction: Aortic valve in diastolic phase (aortic view) $\boldsymbol{D}$. $3 D$ TEE reconstruction: Quantification of the AROA: $0.6 \mathrm{~cm}^{2}$.

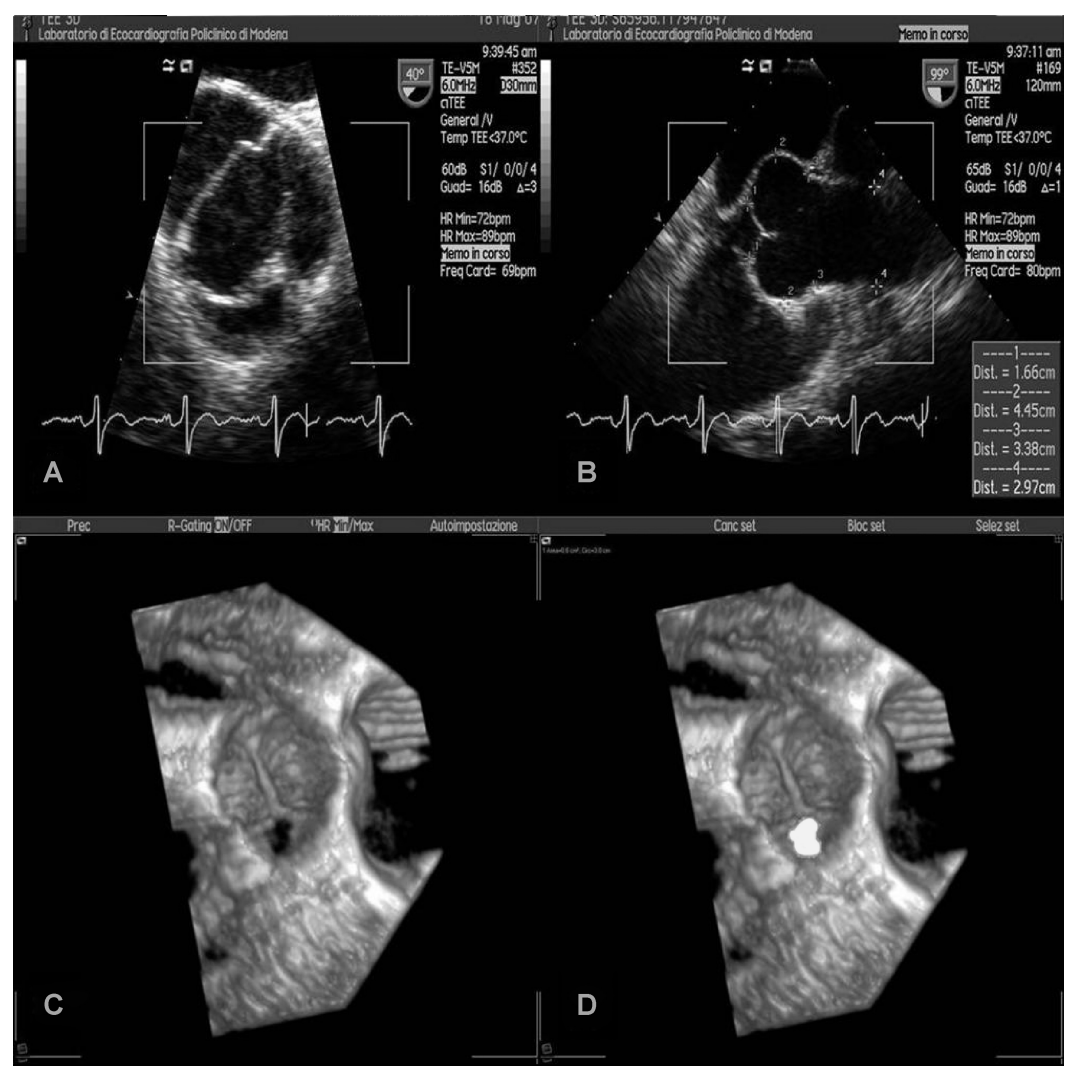


incorporated 3D data acquisition software. In the echocardiography laboratory we performed the offline postprocessing and $3 \mathrm{D}$ reconstruction through a dedicated system (fourSight ${ }^{\mathrm{TM}}$ TEE View, Siemens, USA). By TEE exam we could diagnose a bicuspid aortic valve with fusion of the right and left cusps. The 3D TEE reconstruction allowed to quantify the anatomic regurgitant orifice area (AROA) of the aortic valve. By $2 \mathrm{D}$ TEE, the proximal isovelocity surface area was difficult to measure likely because of the eccentric regurgitant jet. According to the literature ${ }^{1-3} 3 \mathrm{D}$ TEE has limits, including the possibility of creating dropout echoes increasing the AROA as the result of reconstruction rather than real time. Nevertheless, in this patient 3D TEE was superior to $2 \mathrm{D}$ TEE since images are diagnostic.
To the best of our knowledge, few articles $^{4}$ reported AROA's quantification of a bicuspid aortic valve by $3 \mathrm{D}$ reconstruction in an adult patient.

\section{References}

1. Nanda NC, Roychoudhury D, Chung SM, et al: Quantitative assessment of normal and stenotic aortic valve using transesophageal three-dimensional echocardiography. Echocardiography 1994;11:617-625.

2. Rajdev S, Nanda NC, Patel V, et al: Live, real time three-dimensional transthoracic echocardiographic assessment of combined valvar and supravalvular aortic stenosis. Am J Geriatr Cardiol 2006;15:188-190.

3. Ofili EO, Nanda NC: Three-dimensional and fourdimensional echocardiography. Ultrasound Med Biol 1994;20:669-675.

4. Dod HS, Nanda NC, et al: Three-dimensional transesophageal echocardiographic assessment of aortic valve pathology. Am J Geriatr Cardiol 2003;12: 209-213. 